\title{
Frontières
}

\section{Le jury}

\section{Rempart de haute sécurité ou simple muret ?}

\section{Jean-C. Hébert}

Volume 15, numéro 2, printemps 2003

Guerre, mort amère

URI : https://id.erudit.org/iderudit/1073819ar

DOI : https://doi.org/10.7202/1073819ar

Aller au sommaire du numéro

\section{Éditeur(s)}

Université du Québec à Montréal

ISSN

1180-3479 (imprimé)

1916-0976 (numérique)

Découvrir la revue

Citer cet article

Hébert, J.-C. (2003). Le jury : rempart de haute sécurité ou simple muret? Frontières, 15(2), 45-50. https://doi.org/10.7202/1073819ar

\section{Résumé de l'article}

Le jury fait partie des institutions que nous nous sommes données au Canada pour assurer notre sécurité collective. Ce texte examine les trois fonctions du jury : le juge des faits, le promoteur d'équité et le garant de la démocratie. Il aborde également les caractéristiques du système de procès par jury : l'impartialité, la représentativité et la compétence.
Ce document est protégé par la loi sur le droit d'auteur. L'utilisation des services d'Érudit (y compris la reproduction) est assujettie à sa politique d'utilisation que vous pouvez consulter en ligne.

https://apropos.erudit.org/fr/usagers/politique-dutilisation/ 


\section{Résumé}

Le jury fait partie des institutions que nous nous sommes données au Canada pour assurer notre sécurité collective. Ce texte examine les trois fonctions du jury: le juge des faits, le promoteur d'équité et le garant de la démocratie. II aborde également les caractéristiques du système de procès par jury : l'impartialité, la représentativité et la compétence.

Mots clés : jury-procès-justice.

\begin{abstract}
The jury is one of the institutions we have given ourselves in Canada to ensure our collective security. This text examines the three functions of a jury: judge the facts, the promotion of equity and the guarantor of democracy. It also addresses the characteristics of the trial process by jury: impartiality, representativity and competence.
\end{abstract}

Keywords : jury-trial-justice.

\section{LE JURY REMPART DE HAUTE SÉCURITÉ
OU SIMPLE MURET?}

Me Jean-C. Hébert, LL.M.

Avant que le Parlement canadien n'abolisse la peine de mort en $1976^{1}$, dans une affaire de meurtre au premier degré, l'angoisse remuait encore plus profondément les jurés. Puisque la peine de mort sanctionnait un verdict de culpabilité, le spectre d'une erreur judiciaire devenait source de tourment. De nos jours, c'est plutôt l'appréhension de sa propre mort qui peut épouvanter un juré. Pour les membres d'un gang criminalisé traduits en justice, un verdict de culpabilité à leur encontre peut signifier la réclusion perpétuelle. Ne reculant devant rien, ceux-ci peuvent vouloir intimider d'éventuels jurés par la menace de violence ou d'assassinat.

Vénérée par les uns ou critiquée par les autres, l'institution du jury, au passage $\mathrm{du}$ temps, fait voir quelques rides. Soit. S'agit-il pour autant d'un canard boiteux? En matière de criminalité organisée ou de terrorisme, un procès devant jury sert-il les objectifs de la justice pénale? Depuis le retentissant raté du premier procès de dix-sept membres ou sympathisants Hells Angels, certaines voix impatientes réclament rien de moins qu'une justice d'exception. Plutôt que sa mise à l'écart, vaut-il mieux étançonner l'institution du jury en neutralisant les causes d'intimidation? Ces interrogations, au demeurant fort légitimes, exigent l'examen des fonctions et caractéristiques du système de procès par jury.

\section{LES FONCTIONS DU JURY}

Pour la Cour suprême du Canada, la plupart des raisons historiques mises de l'avant pour justifier le procès par jury se révèlent aussi pertinentes aujourd'hui qu'elles l'ont été au cours des siècles. Cependant, la modernité s'accompagne d'autres justifications ${ }^{2}$. Comme juge des faits, le jury serait parfois un meilleur arbitre judiciaire que la magistrature. Sa propension à juger en équité en ferait la conscience de la collectivité. De plus, le jury serait une garantie de démocratie ou une sorte de rempart contre l'oppression des lois. Ces trois fonctions méritent un instant de réflexion.

\section{LE JUGE DES FAITS}

En général, la diversité des membres d'un jury et l'expérience de vie font de cet arbitre communautaire un meilleur juge des faits que le magistrat isolé (Commission de réforme du droit, 1980 , p. 6). Cette proposition vaut certainement quant à l'appréciation de la crédibilité des témoins. Puisque la plupart des procès portent sur la détermination des inférences étayées par la preuve $^{3}$, la mémoire collective du jury lui confère un net avantage dans le processus d'examen de la responsabilité pénale de l'inculpé (Commission de réforme du droit, 1980, p. 6). Enfin, la délibération de groupe garantit un examen du dossier sous toutes ses coutures. Le débat oral neutralise la tendance à tenir pour avérés certains faits litigieux (Commission de réforme du droit, 1980, p. 6-7). Récemment, la Cour suprême a redit les avantages du processus de délibération collective propre au jury et rappelé le caractère essentiel de cet organe judiciaire du processus pénal ${ }^{4}$.

Sans nier les vertus reconnues au jury comme juge des faits, certaines remarques doivent moduler la description qui précède. Curieusement, ce n'est qu'après avoir entendu l'ensemble de la preuve que les 
jurés sont informés des éléments juridiques encadrant leur fonction de juge des faits. Connaisseur du droit, le juge dispose parfois d'un certain avantage sur le jury pour soupeser les témoignages puisqu'il peut focaliser son attention sur les passages importants dès leur insertion dans les cahiers de la preuve (Jackson et Doran, 1997, p. 773). Mal renseigné sur les concepts juridiques pertinents, le jury peut certes éprouver des difficultés à capter l'intelligence d'une preuve administrée en pièces détachées, selon le bon vouloir des parties.

D'aucuns estiment qu'un magistrat serait plus compétent qu'un jury pour évaluer certains types de preuve. Ainsi, les questions d'identification et les débats d'ordre scientifique seraient mieux compris par un juge expérimenté (Jackson et Doran, 1997, p. 772). Rappelons pour mémoire que la pertinence ou la simple utilité d'une preuve d'expert ne suffit pas pour la rendre admissible. Elle doit plutôt être essentielle à l'évaluation des faits. Lorsque la technicité d'un élément de preuve brouille les pistes, un décideur doit pouvoir se former une opinion juste sur une affaire avec l'aide indispensable de personnes dotées de connaissances particulières $^{5}$. Le critère de nécessité tient au fait que, risquant d'être subjugués par le discours d'un témoin savant, les jurés seraient plus enclins à suivre servilement son opinion. Pour faire bonne mesure, reconnaissons que bien des juges réagissent de la même façon devant un témoignage d'expert.

\section{LE PROMOTEUR D'ÉQUITÉ}

N'étant pas tenu de motiver son verdict, le jury peut instiller une bonne dose d'équité dans la conduite d'un procès pénal. Sans écorner la vocation de la loi pénale, les jurés peuvent néanmoins apprécier avec plus de flexibilité qu'un juge seul l'écart de conduite reproché à l'accusé dans un contexte bien précis (Holdsworth, 1922, p. 349). S'agissant d'apprécier le bien-fondé d'une inculpation, la magistrature doit voir à l'application de la loi. Imputable devant la société, le juge fonde son analyse sur les concepts et standards émanant du droit positif ou prétorien. Au contraire, incarnant la collectivité, le jury peut mettre l'accent sur certaines valeurs communautaires dans l'exercice de sa mission ${ }^{6}$. Sous réserve de rendre un verdict raisonnable, les jurés n'ont de compte à rendre à personne (Jackson et Doran, 1997, p. 764).

Gardons-nous de conclure hâtivement que la rectitude du jugement prononcé par un magistrat serait supérieure à celle du verdict rendu par un jury. Afin de ratisser large, la loi pénale s'exprime souvent en termes généraux. Régissant des cas individuels, son application exige souplesse et nuance afin de garantir à l'inculpé, au plaignant et aux témoins l'équité du processus judiciaire. D'ailleurs, la constitution garantit formellement à tout citoyen le droit inaliénable d'avoir un procès équitable ${ }^{7}$. Certes, l'application rigide d'un texte d'incrimination n'est pas exempt d'injustice. Conscience de la collectivité, le jury peut occasionnellement juger équitable la relaxe d'un accusé (Commission de réforme du droit, 1980, p. 10-11). Ainsi, lors d'une inculpation de fraude axée sur la preuve d'un risque de préjudice économique, plutôt que sur la démonstration d'une véritable perte, les jurés peuvent apprécier l'exigence de conduite malhonnête favorablement à l'inculpé.

Chaque fois que l'analyse de la culpabilité passe par le prisme d'un standard d'appréciation, contrairement à la magistrature, les jurés ne sont pas distraits par le vernis d'uniformité propre au mimétisme jurisprudentiel. Dans la lorgnette d'un jury, l'acte raisonnable, l'écart marqué d'une inconduite, la moralité d'un geste, la connaissance coupable (et son excroissance qu'est la cécité volontaire), la tolérance, l'insouciance et l'intention criminelle sont autant d'éléments faisant appel à des valeurs communautaires susceptibles de déborder les balises étroites observées par la magistrature (Jackson et Doran, 1997, p. 766). Principe de justice fondamentale, le droit à un procès équitable est celui qui permet au juge des faits de découvrir la vérité et de rendre une décision équitable ${ }^{8}$. Pour ce faire, le jury jouit indubitablement d'une large discrétion judiciaire. La pesée de différents impératifs oblige le juge des faits à s'assurer que l'équilibre recherché est en phase avec le but ultime de sa mission, soit la tenue d'un procès parfaitement équitable.

\section{LE GARANT DE LA DÉMOCRATIE}

Le jury constitue indubitablement une garantie de démocratie. Voilà pourquoi la société respecte grandement les verdicts rendus par un segment de la communauté. La justice en direct semble plus légitime que l'appel au juge rendant jugement au nom de la collectivité (Jackson et Doran, 1997, p. 771). Le jury incite les tribunaux d'appel à l'exercice d'une prudente retenue lors d'une allégation de verdict déraisonnable ${ }^{10}$. L'indépendance de la magistrature reste un concept mal compris de la société civile. Cette avancée dans l'affirmation du principe cardinal de la séparation des pouvoirs laisse plutôt froide et sceptique l'opinion publique, laquelle perçoit fréquemment le juge comme un joueur gouvernemental.

Au contraire, les jurés jouissent d'une image d'absolue indépendance face à l'État. Cette perception alimente un sentiment favorable au jury, notamment lorsqu'il s'agit de passer en jugement des personnes accusées de crimes graves. Bref, dans une salle d'audience, les jurés sont perçus comme le matériau externe arrimé à la fibre composite du tribunal (Jackson et Doran, 1997, p. 760). En somme, l'avantage du procès par jury ne réside pas tellement dans la capacité des jurés de toujours mieux apprécier les faits. Il réside surtout dans une situation, tout aussi réelle qu'apparente, d'indépendance du tribunal face à l'État accusateur (Jackson, 1996, p. 90). En son temps, Tocqueville fut d'avis que le jury était porté à percevoir le juge comme l'instrument passif du pouvoir social et, dans une certaine mesure, à se méfier de ses avis (Tocqueville, 1848, p. 408). À notre avis, ce germe de méfiance existe toujours. Il pourrait même trouver un terreau fertile dans les cas de poursuites teintées d'abus de pouvoir ou fondées sur des incriminations impopulaires (King, 1999, p. 51). Dans un tout autre contexte, cette méfiance du corps des jurés pourrait s'aviver dans l'hypothèse où les subordonnés de l'État feraient un usage musclé des pouvoirs d'exception prévus en matière de criminalité organisée et de terrorisme (King, 1999, p. 51) ${ }^{11}$.

Le jury est rien de moins qu'un organe judiciaire du processus pénal. Dans une large mesure, il exerce le même rôle que les magistrats siégeant sans jury. Dans un procès aux assises criminelles, le juge et le jury produisent le jugement de la cour. Afin de parvenir à un verdict, les jurés appliquent le droit aux faits ${ }^{12}$. Depuis l'enchâssement de la Charte canadienne des droits et libertés dans la constitution, le pouvoir judiciaire doit exercer un nouveau rôle : la défense des libertés individuelles fondamentales et des droits de la personne contre les ingérences de l'État ${ }^{13}$. Procédant à l'application du droit aux faits, le jury peut légitimement et légalement sanctionner les abus de l'État quelle qu'en soit la forme. Ici, le concept de droit vise la définition d'une infraction, la description d'une norme de preuve, l'application d'une règle de procédure et le respect d'un droit fondamental garanti par la constitution. Gardant à l'esprit les observations qui précèdent, voyons brièvement quel est l'état du droit canadien sur la délicate question du pouvoir d'un jury d'annuler la portée d'une loi par le biais du verdict rendu.

La plus haute cour du pays aborda le sujet dans l'affaire Morgentaler ${ }^{14}$. Dans sa plaidoirie, le procureur de la défense appelait le jury à ne pas appliquer le texte d'incrimination en matière d'avortement s'il considérait la loi mauvaise. Ce faisant, il signalait au Parlement la nécessité de changer la $10 i^{15}$. Cosignataires d'une opinion conjointe, approuvée par leurs collègues, les juges Dickson et Lamer ont vigoureusement désapprouvé l'initiative du procureur de la défense. Cette singulière démarche serait de 
nature à engendrer de graves iniquités. La Cour disait craindre une justice d'humeur et la disparité des verdicts. De surcroît, dans une société pluraliste, l'action débridée du jury pourrait donner ouverture à un verdict orienté par les passions du racisme ${ }^{16}$. Cela dit, les juges Dickson et Lamer élaborèrent une solution de compromis : bien qu'aucun droit n'autorise le jury à le faire, il possède néanmoins le pouvoir d'ignorer l'enseignement du droit dispensé par le juge. Puisqu'il ne s'agit pas d'un droit, le procureur de la défense ne peut en faire état dans sa plaidoirie ${ }^{17}$. La faiblesse évidente de cette approche réside dans le fait que le jury ignore l'existence d'un important pouvoir sans égard au poids de la preuve. Pour la Cour, d'utilisation rarissime, ce concept inusité pourrait s'expliquer par l'oppression découlant d'une loi sévère ou de son application stricte ${ }^{20}$. Abordant l'examen de la légitimité de ce pouvoir du jury sous l'angle de la logique et des principes, la plus haute cour du pays affirme péremptoirement que l'annulation de l'effet de la loi est antinomique au droit. En fait, ce pouvoir ne serait rien d'autre qu'un fait, c'est-à-dire un acte d'insoumission ou de protestation. Et, d'ajouter la Cour, la tolérance d'un fait ne peut aucunement justifier l'encouragement à la désobéissance civile par un plaideur ou par un juge dans son exposé au jury ${ }^{21}$.

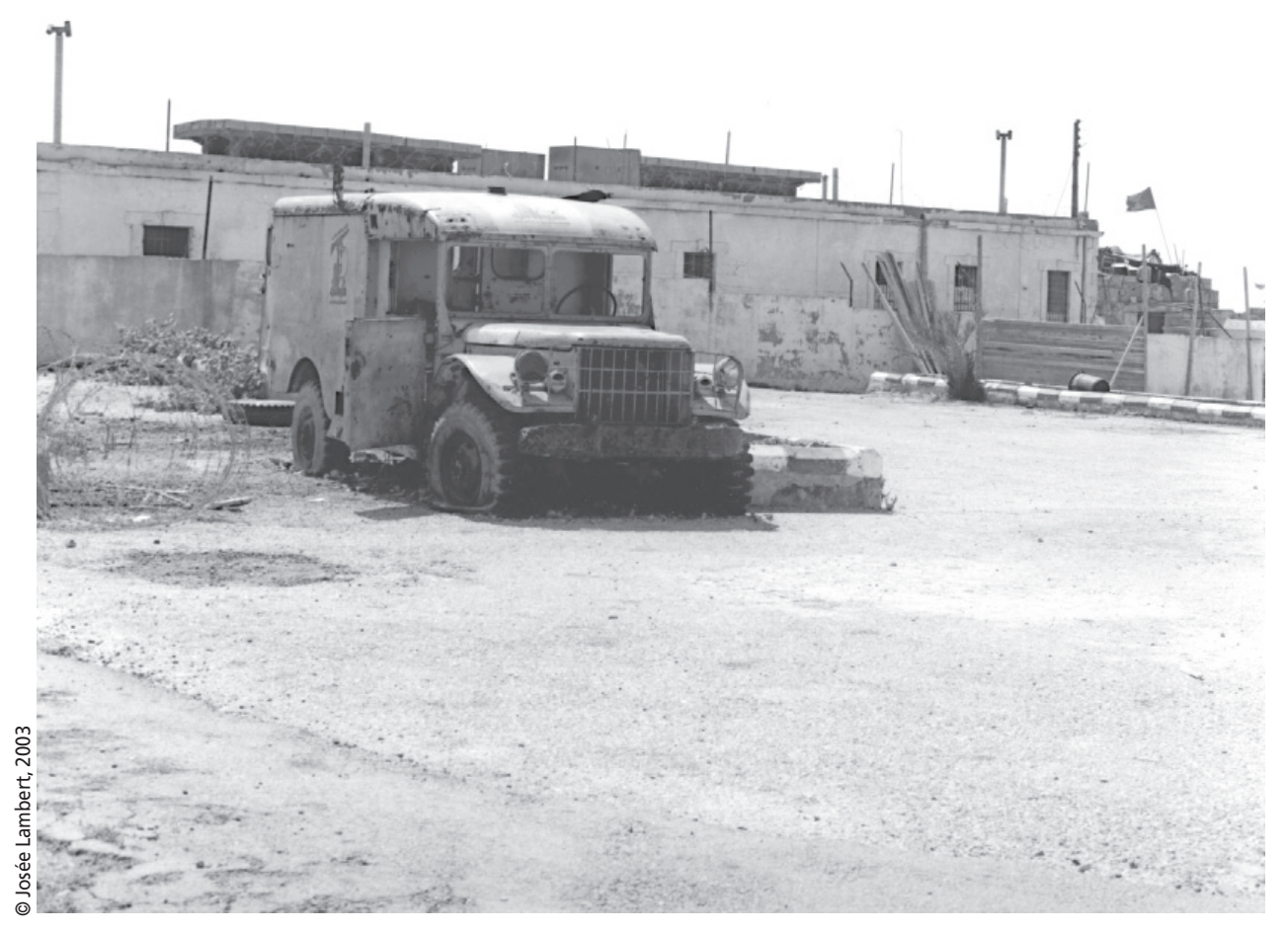

et ne dispose d'aucune balise pour l'exercer. Ironiquement, en acquittant le docteur Morgentaler et ses associés parce qu'il réprouvait la loi, le jury a devancé le jugement de la Cour suprême sanctionnant l'inconstitutionnalité du texte d'incrimination ${ }^{18}$.

La question du pouvoir d'annulation du jury refit surface dans l'affaire Latimer $^{19}$. Le jugement unanime de la Cour suprême fut rendu per curiam. On y précise d'abord le sens de l'expression «annulation par le jury ». Il s'agit d'un situation rare où le jury choisit, en connaissance de cause, de ne pas appliquer la loi et acquitte l'inculpé

\section{Les survivants, les victimes fuient dans l'absence et le silence.}

Puisque le procureur de Latimer plaidait que le pouvoir d'annulation du jury avait été miné par le magistrat de première instance, il alléguait une atteinte au droit de son client d'avoir un procès équitable. Cet argument fut sèchement rejeté. Pour la Cour, l'appelant ne pouvait légitimement invoquer un droit général à l'annulation par le jury, puisqu'il ne s'agit pas d'un élément valable. Par conséquent, le procès ne pouvait être inéquitable du seul fait que le juge du procès avait miné le pouvoir d'annulation du jury. Poussant plus avant la réflexion, la Cour statua qu'il est légitime et souhaitable qu'un magistrat empêche l'annulation par le jury. Dite autrement, cette proposition signifie que le juge doit prendre les mesures nécessaires pour que le jury applique la loi correctement ${ }^{22}$.

Pour l'heure, l'état du droit canadien concernant le pouvoir d'annulation du jury semble bien circonscrit. D'une part, la magistrature doit s'évertuer à convaincre le jury d'appliquer la loi dans toute sa rigueur. D'autre part, les plaideurs sont bâillonnés quant à la possibilité d'inviter le jury à protester contre les abus de l'État quant à l'adoption et à l'application des lois en acquittant l'inculpé. Ce jugement sonne le glas au pays de la théorie juridique faisant du jury le « rempart» contre l'oppression des lois. La tolérance judiciaire du «fait » de l'annulation de la loi nous oblige désormais à considérer le jury comme un « muret» protecteur de la servitude des lois. Rappelons pour mémoire que c'est ce rôle de champion de la liberté et de protecteur ultime des citoyens contre l'application arbitraire de la loi ou l'oppression étatique qui a mérité à l'institution du jury les plus vibrants éloges (Commission de réforme du droit, 1980, p. 11).

D'utilisation rarissime, le pouvoir d'annulation n'en conserve pas moins une importance capitale dans une société démocratique. La résonance médiatique contraint inévitablement les autorités publiques à prendre en compte l'appel communautaire dénonçant la conduite abusive des agents de l'État ou suggérant la révision d'une loi ébréchée par le verdict unanime de douze citoyens (Commission de réforme du droit, 1980, p. 12-13). Certes, on ne peut jamais exclure la possibilité d'égarement passager d'un jury. Cependant, l'expérience démontre que, dans la plupart des cas, les jurés se font un devoir d'appliquer la loi conformément à l'enseignement du juge. Dans une perspective d'équilibration, les avantages pour la société de l'insubordination du jury l'emportent substantiellement sur les dangers susceptibles d'apparaître (Commission de réforme du droit, 1980, p. 13).

Si l'appel à la mutinerie contre la loi se conçoit plutôt mal dans une cour de justice, la contestation de son application nous est familière. Nous savons d'ores et déjà que la magistrature s'est autoproclamée défenseur des droits et libertés, d'abord par la reconnaissance de la doctrine d'abus de procédure, ensuite par l'élargissement de sa mission constitutionnelle. En cette matière, un débat judiciaire se tient en toute 
transparence. À l'opposé, s'agissant du « fait» ou « pouvoir » d'annulation reconnu au jury, la Cour suprême privilégie l'opacité du non-dit. Ainsi, des jurés, renseignés (par les médias) sur leur compétence, pourraient désapprouver la teneur d'une disposition législative ou censurer l'iniquité d'une poursuite. Par contre, d'autres jurés, ignorant leur capacité d'action, risquent de comprendre tardivement le retentissement social d'un verdict. Dans l'affaire Latimer, un juré déclara publiquement qu'il aurait voté pour l'acquittement (d'un père en détresse convaincu de meurtre par compassion) s'il avait su que l'inculpé encourait l'emprisonnement à perpétuité.

Ce double standard remue le sens de justice et rappelle le bandeau sur les yeux de Thémis. Martin Luther King disait d'un citoyen, brisant une loi injuste afin d'éveiller la conscience collective, qu'il manifeste en vérité un grand respect pour la Loi. À dire vrai, le rôle des jurés n'est pas d'appliquer mécaniquement la loi mais plutôt de rendre justice dans un environnement où règne la primauté du droit. Au terme d'un examen de la preuve effectué sous l'éclairage du droit, dire ce qui est juste ou injuste oblige le juge des faits à prendre en compte les valeurs communautaires et l'appel de sa conscience. Le magistrat présidant un procès aux assises criminelles peut, dans l'exercice de sa vaste discrétion judiciaire, relaxer les règles de preuve ${ }^{23}$ afin de permettre à l'accusé d'enrichir le fondement factuel de son argumentation contre l'oppression de la loi ou l'iniquité d'une poursuite. Un juge peut donc, en toute légalité, permettre au jury d'être mieux renseigné quant à l'impact social de son verdict.

Sans encourager explicitement l'exercice du pouvoir d'annulation du jury, un juge peut tout de même éviter de l'obscurcir au point de favoriser son éradication. Dans une société démocratique, sous le regard scrutateur de l'opinion publique, le corps judiciaire doit être en harmonie avec le corps social. C'est le moment de rappeler que l'institution du jury comporte une facette d'éducation collective favorisant une perception favorable dans l'opinion publique du système de justice pénale ${ }^{24}$.

\section{LES CARACTÉRISTIQUES DU JURY}

L'impartialité, la représentativité et la compétence du jury sont les principales caractéristiques mises de l'avant par plusieurs opinions judiciaires ou doctrinales. L'exigence d'impartialité ne souffrant d'aucune discussion, le débat porte plutôt sur la façon d'atteindre cet objectif. La question de représentativité pose avec acuité le problème de la définition de cette exigence. Enfin, s'agissant de la compétence des jurés, cette caractéristique revêt une dimension particulière lors de procès de longue durée portant sur des matières complexes.

\section{L'IMPARTIALITÉ}

Lorsqu'un juré est sur le point de prêter serment, l'importance du mécanisme de récusation motivée prend tout son relief puisqu'il permet de se renseigner sur son état d'esprit. Par conséquent, le juge du procès ne saurait user de son pouvoir discrétionnaire pour restreindre ce droit ${ }^{25}$. En cas de doute, le juge doit pécher par excès de prudence $^{26}$ et permettre la demande de récusation pour empêcher le jury d'être composé de membres partiaux. Par contre, l'exercice de ce droit bascule dans l'illégitimité dès lors qu'on y recourt pour fausser la représentativité du jury ou pour obtenir abusivement des renseignements personnels sur un candidat ${ }^{27}$.

L'accusé peut vouloir récuser un candidat au poste de juré lorsqu'il y a une possibilité réaliste que celui-ci entretienne un préjugé faussant son impartialité. Linterrogatoire sert à décider si la personne choisie au hasard sera capable d'agir impartialement. La Cour suprême, dans l'arrêt $W_{i l l i a m s}{ }^{28}$, a indiqué que l'inclination à la partialité d'un candidat peut provenir de l'une ou l'autre des catégories suivantes : le préjugé fondé sur un intérêt direct dans le procès $^{29}$; le préjugé spécifique lié à des attitudes ou croyances concernant la cause ${ }^{30}$; le préjugé générique prenant appui sur des attitudes stéréotypées à propos de l'accusé, les plaignants, les témoins ou en raison de la nature du crime allégué ${ }^{31}$; enfin, le préjugé inspiré par le conformisme, c'est-à-dire une pression sociale suscitant une attente quant à l'issue de la cause.

La plus haute cour du pays devait subséquemment préciser, dans l'arrêt Find ${ }^{32}$, que deux conditions sont exigées pour établir une possibilité réaliste de partialité au sein des jurés. L'une a trait à l'attitude, c'està-dire à l'existence d'un préjugé largement répandu au sein de la collectivité; l'autre concerne le comportement, c'est-à-dire que, malgré des garanties procédurales, certains jurés pourraient être incapables de surmonter un préjugé. C'est le moment de rappeler que le jury n'a pas à être immunisé contre toute forme de préjugé. À titre d'exemple, notre système de justice pénale lui impose d'avoir un préjugé favorable envers le principe de justice fondamentale de la présomption d'innocence et ses attributs procéduraux (Gobert, 1988, p. 326).

Reprenons l'un et l'autre concepts. Sous l'angle de l'attitude, un préjugé est un parti pris en faveur ou à l'encontre d'une chose, d'une personne ou d'un groupe par rapport à un autre, particulièrement d'une manière jugée injuste. Dans le contexte d'une récu- sation motivée d'un candidat juré, le concept de préjugé s'entend d'une attitude susceptible d'amener des jurés à remplir leur rôle d'une manière préjudiciable et injuste. Diverses attitudes émotives ou stéréotypées ne constituent pas toutes des préjugés. Le préjugé doit pouvoir influer injustement sur l'issue d'une cause. L'existence d'un préjugé est déterminée dans le contexte d'une affaire. Il doit être largement répandu dans la collectivité. Un préjugé peut naître de plusieurs circonstances : existence d'un intérêt personnel; connaissance préalable de la cause en raison de sa médiatisation; la race de l'accusé; nature et circonstances de l'infraction alléguée. Sous l'angle du comportement, l'impartialité du jury n'est pas synonyme de neutralité. Un juré n'a pas à faire abstraction de ses opinions, croyances, connaissances et autre bagage d'expériences. Le vécu des jurés éclaire leur délibération. La diversité est essentielle à leur tâche de décideur collectif et de conscience représentative de la collectivité. La diversité de point de vue fait partie de l'institution du jury, d'où le fait que leur verdict reflète les valeurs communes de la collectivité.

Il n'y a pas que les récusations motivées qui sont susceptibles de garantir à l'inculpé la tenue d'un procès équitable, par le biais de la formation d'un jury impartial ${ }^{33}$. Dans une certaine mesure, le jeu des récusations péremptoires $^{34}$ permet aux parties de façonner le profil du jury. L'exercice de ce droit ne doit jamais servir à neutraliser l'exigence de représentativité des jurés conscrits. Toutefois, ce mécanisme arbitraire d'élimination de certains candidats peut, à la limite, améliorer le caractère représentatif d'un jury. D'ailleurs, la Cour suprême a reconnu l'aspect entièrement subjectif d'une récusation péremptoire ${ }^{35}$. Pour tout dire, il s'agit bel et bien d'une contribution partisane à la recherche d'un jury impartial ${ }^{36}$. Enfin, la participation active de l'accusé au choix de ses pairs est de nature à rasséréner sa confiance quant à l'impartialité du tribunal.

Les différents filtres prévus par la loi pour sélectionner des candidats jurés impartiaux ne sont pas totalement étanches. Par conséquent, certaines personnes choisies peuvent ressentir, plus ou moins secrètement, des élans de partialité. Le procès équitable devant un «tribunal » impartial signifie que l'accusé ne peut revendiquer le droit à un procès devant douze jurés affichant tous le même niveau d'impartialité. La somme d'impartialité du jury peut être plus grande que l'objectivité de certains de ses membres. Les juges étant censés respecter leur serment ${ }^{37}$, cette présomption vaut également pour les jurés. En principe, la procédure encadrant le procès pénal permet de neutraliser les préjugés, opinions ou 
croyances des jurés. D'ailleurs, l'exposé initial du juge et des procureurs souligne aux jurés le sérieux de leur tâche et les enjoint de faire montre d'objectivité. Enfin, les règles de preuve et de procédure les dirigent vers l'appréciation de la preuve et du droit pertinent. Pour la Cour suprême ${ }^{38}$, ces puissants antidotes contre les émotions, les idées préconçues et les préjugés garantissent l'équité du procès.

L'impartialité ne peut se concevoir en demi-portion. Alors que l'indépendance d'un tribunal peut être conçue sous forme de continuum, la notion d'impartialité repose sur une dichotomie entre deux états d'esprit: la partialité ou la non-partialité. En somme, l'impartialité d'une cour de justice devrait être irréprochable ${ }^{39}$. Voilà pour la théorie juridique. En pratique, l'impartialité reste un idéal à atteindre. Transposé dans un cas de figure, ce concept devient relatif. Sous l'angle de la discussion, ce n'est pas nier l'idéal d'impartialité que d'admettre, selon les circonstances, qu'il puisse exister à des degrés variables.

\section{LA REPRÉSENTATIVITÉ}

Appelé à présider un long procès de meurtre impliquant plusieurs accusés, un juge afficha publiquement son désir de recourir à son pouvoir discrétionnaire ${ }^{40}$ d'exempter les candidats susceptibles d'encourir un préjudice financier par l'exercice de la fonction de juré ${ }^{1}$. Cette manifestation de bonne intention signifie qu'un important segment de la société peut être dispensé du devoir civique de rendre justice. Dans la mesure où des professionnels, des gens d'affaires ou des travailleurs autonomes sont exclus d'un jury lors d'un procès de longue durée, la typicité de l'institution risque fortement d'être carencée. L'hypothèse, bien réelle, qu'un jury puisse majoritairement être composé de retraités, de chômeurs ou d'assistés sociaux entretient l'inquiétude. Face à cette réalité, il est impérieux de voir comment les tribunaux traitent l'exigence de représentativité du jury.

C'est dans l'arrêt Sherratt ${ }^{42}$ que la Cour suprême a, pour la première fois, abordé le sujet en indiquant que la garantie constitutionnelle du droit au procès par jury repose sur deux fondements importants: l'impartialité et la représentation maximale de la société. L'importance accordée au caractère représentatif du jury résulte non seulement de l'exigence d'un procès équitable pour le citoyen inculpé mais aussi du droit ou du devoir de ses concitoyens d'être membres du jury. Des juges tracent parfois une adéquation entre la capacité des jurés de prendre en considération les valeurs communautaires et leur diversité d'origine ${ }^{43}$. D’où, la nécessité pour le jury de représenter toutes les couches de la société. Certains estiment que le mécanisme de récusation motivée ou péremptoire a notamment pour objet d'assurer cette qualité essentielle d'un jury qu'est sa représentativité. D'autres, au contraire, pensent que la démarche des procureurs au niveau de la sélection des jurés a pour effet, sinon pour objet, d'affaiblir l'idéal de représentativité ${ }^{44}$.

Dans l'arrêt Bain ${ }^{45}$, effleurant la question de représentativité dans son opinion dissidente, le juge Gonthier a rappelé les observations faites par la juge L'HeureuxDubé dans l'affaire Sherratt. Subséquemment, dans l'arrêt Biddle ${ }^{46}$, les juges majoritaires ont évité d'aborder le sujet de la représentativité du jury au motif qu'il s'agissait d'une question académique. Au contraire, rédigeant une opinion minoritaire concordante, le juge Gonthier crut nécessaire de développer sa pensée sur le sujet. Pour lui, la représentativité est une caractéristique qui, sans la garantir complètement, favorise la perception d'impartialité. Bien qu'il ne s'agisse pas d'une qualité essentielle d'un jury, il faut néanmoins la rechercher. La véritable garantie d'impartialité du jury dépend du couplage de la représentativité et de l'exigence d'unanimité du verdict des jurés. Conséquemment, toute tentative de modifier la composition du jury en diluant sa représentativité constitue une atteinte à son impartialité 47 .

Bien qu'elle ait antérieurement souscrit à la dissidence du juge Gonthier dans l'arrêt Bain, la juge McLachlin marqua son désaccord avec la position de son collègue. Si un jury doit être impartial et compétent, la loi n'exige pas qu'il soit représentatif. L'actuelle juge en chef du pays brandit un argument historique pour étayer sa thèse : l'impossibilité de conclure rétroactivement à l'impartialité des jurys qui, pendant des siècles, furent composés exclusivement d'hommes. Favorable à la représentativité du jury, la juge McLachlin écarte néanmoins l'idée que ce soit une finalité de la loi. Cette norme lui semble impossible à atteindre. L'atomisation de la société en classes et sous-classes par le jeu de caractéristiques comme le sexe, la race, l'instruction et la profession rend utopique l'atteinte d'une représentativité idéale. Celle-ci ne peut être qu'un moyen d'atteindre l'impartialité et la compétence du jury, de conclure la juge McLachlin. Ce dernier argument est plus convaincant que la référence historique illustrant la discrimination faite aux femmes. Peut-on corriger une erreur ancienne par l'acceptation d'une représentativité qui serait singularisée selon le sexe des jurés?

Pour la composition d'un jury, notre système de justice pénale recherche la diversité de points de vue et d'opinions. Un brassage d'idées par des jurés venant de milieux différents devrait favoriser un débat énergique et complet. Il ne faut pas confondre diversité et géographie. La répartition de groupes culturels dans différentes localités ne garantit aucunement la diversité de points de vue. Curieusement, l'objectif d'impartialité du jury sera mieux servi par l'acceptation des préjugés caractérisant certains groupes sociaux. L'affichage des idées préconçues de l'un permet à l'autre de les répudier et viceversa. Après la mise à l'écart des stéréotypes et des visions dogmatiques, la dynamique de groupe facilite l'atteinte des valeurs communautaires facilitant l'analyse du cas d'espèce faisant l'objet du procès.

\section{LA COMPÉTENCE}

Le jury mérite la confiance du public. Depuis des centaines d'années, il applique les règles de droit en fonction du bon sens contemporain $^{48}$. Le sens commun reflète les perceptions collectives. Celles-ci sont largement considérées comme des faits par les citoyens. À ce titre, elles font partie de nos valeurs $^{49}$. Une forte présomption de compétence caractérise l'institution du jury. Disposant de directives claires et précises, les jurés sont ordinairement en mesure de bien remplir leur mission ${ }^{50}$, d'où le principe d'inclusion de la preuve. Mieux vaut s'en remettre au bon sens des jurés et leur donner tous les renseignements pertinents. Cette assertion vaut également pour les cas où le procès porte sur une matière confuse. Il est fréquent qu'un procès soulève des questions de preuve complexes. Bien que la simplicité ait ses vertus, il est impératif qu'un jury dispose de toutes les informations utiles à l'exercice de son mandat ${ }^{51}$. L'exposé au jury doit permettre aux jurés de comprendre leur rôle comme juge des faits et de saisir les éléments essentiels de l'affaire à juger.

Postulons qu'un juge peut comprendre une preuve massive et embrouillée. Avec l'aide des procureurs, il devrait être en mesure de l'expliquer au jury. D'ailleurs, notre système de justice pénale accorde une très grande confiance à la capacité des jurés de suivre les directives du juge ${ }^{52}$. Même dans un climat de confusion, il ne faut pas conclure que les jurés seraient aussi benêts qu'un magistrat engourdi. Hélas, le bon ton de ce discours ne suffit pas pour dissiper le scepticisme manifesté par plusieurs intervenants de la classe juridique quant à la capacité des jurés de rendre justice dans certaines affaires.

Le juge Gonthier a même avancé l'idée que la complexité d'un procès pourrait justifier une entorse au mécanisme de sélection au hasard d'un jury afin d'assurer une aptitude minimale de compréhension de la preuve et des questions en litige ${ }^{53}$. Cette étonnante proposition met en cause le degré de compétence requis pour être juré. Le sens commun d'une personne ne 
se mesure pas à la nature, la durée ou la qualité de sa formation académique. Tolérer qu'un juge puisse user de son pouvoir discrétionnaire ${ }^{54}$ pour débusquer les ignares porterait atteinte à la dignité des candidats jurés sélectionnés au hasard. De surcroît, laissant une large place à l'arbitraire et porteuse d'un germe discriminatoire, l'exercice d'une telle discrétion serait potentiellement inconstitutionnelle. Il revient aux parties, par le biais de la récusation, d'écarter les candidats jurés dont ils appréhendent l'incompétence.

Les abolitionnistes du jury invoquent l'argument de complexité. Ainsi, les questions économiques et financières seraient à ce point compliquées que des jurés risquent d'être confondus. Cette thèse élitiste ignore la capacité d'une personne raisonnable de comprendre la malhonnêteté d'une conduite. Pour ce faire, nul besoin que le jury soit versé dans le domaine de la quincaillerie financière. Une gestion efficace du procès en général et de l'administration de la preuve en particulier peut grandement faciliter la compréhension des jurés. En soi, la durée du procès n'est pas un facteur d'incompréhension (Findlay, 2001, p. 73).

Lidentité des jurés étant de nos jours mieux protégée que jamais et les mesures de sécurité étant substantiellement accrues, l'appréhension d'un juré de subir un mauvais sort s'en trouve autant diminuée. Il coule de source que l'appréhension de la mort chez un juré appelé à juger de dangereux criminels ne constitue certes pas un facteur justifiant le législateur de changer la donne.

\section{Bibliographie}

CASEY, A. R. (1991). Justice on Trial: Report of the Task Force on the Criminal Justice System and Its Impact on the Indian and Metis People of Alberta, Edmonton, ministère du Solliciteur général de l'Alberta.

COMMISSION DE RÉFORME DU DROIT (1980). Le jury en droit pénal, Document de travail 27.

FINDLAY, Mark (2001). "Juror comprehension and complexity», Brit. J. Criminol, vol. $41, \mathrm{n}^{\circ} 56$.

GOBERT, James J. (1988). «In search of the impartial jury», Journ. Crim. Law \& Criminology, vol. 79, n ${ }^{\circ} 269$.

HAMILTON, A. C. et C. M. SINCLAIR (1991). Report of the Aboriginal Justice Inquiry of Manitoba, Winnipeg, vol. 2.

HOLDSWORTH, W. (1922). A History of English Law, 3e éd., Londres, Methuen.

JACKSON, John (1996). "Judicial responsibility in criminal proceedings ", Current Leg. Prob., vol. 49, n 59.

JACKSON, John et Sean DORAN (1997). "Judge and jury: Towards a new division of labour in criminal trials", Mod. L.R., $\mathrm{n}^{\circ} 759$.
KING, Nancy Jean (1999). «The American criminal jury», Law \& Contemp. Probs., vol. $62, \mathrm{n}^{\circ} 41$.

TOCQUEVILLE, Alexis de (1848). De la démocratie en Amérique, Tome I, Paris, Gallimard.

\section{Notes}

1. Ce n'est qu'en 1998 que le législateur canadien a définitivement aboli la peine de mort qui subsistait encore dans le Code de discipline militaire.

2. R. c. Sherratt, [1991] 1 R.C.S. 509, p. 523.

3. R. c. Rose, [1998] 3 R.C.S. 262, par. 22 (j. Binnie pour l'opinion dissidente).

4. R. c. Pan; R. c. Sawyer, [2001] 2 R.C.S. 344, par. 41 à 43.

5. R. c. D.D., [2000] 2 R.C.S. 275, par. 46-47.

6. R. c. Lessard, (1992) 74 C.C.C. (3d) 552, p. 559.

7. L'alinéa $11 d$ ) de la Charte canadienne des droits et libertés dispose que tout inculpé a le droit d'être passé en jugement dans le cadre d'un procès public et équitable.

8. R. c. Seaboyer, [1991] 2 R.C.S. 577, p. 608.

9. R. c. Underwood, [1998] 1 R.C.S. 77, par. 6.

10. R. c. E.(A.W.), [1993] 3 R.C.S. 155, p. 193 (j. Cory pour l'opinion majoritaire).

11. En Grande-Bretagne, à la suite d'un rapport rédigé par Lord Diplock, le droit au procès par jury fut aboli en matière d'accusations liées au terrorisme.

12. R. c. Pan; R. c. Sawyer, [2001] 2 R.C.S. 344, par. 43.

13. R. c. Beauregard, [1986] 2 R.C.S. 56, p. 72.

14. R. c. Morgentaler, [1988] 1 R.C.S. 30.

15. Id., p. 76.

16. Id., p. 77.

17. Id., p. 78-79.

18. Id., p. 126.

19. [2001] 1 R.C.S. 3.

20. Id., par. 57.

21. Id., par. 68 .

22. Id., par. 70 .

23. R. c. Brown, 2002 CSC 32, par. 42. Un tribunal a le pouvoir discrétionnaire résiduel d'assouplir en faveur de l'accusé une règle de preuve stricte lorsque cela est nécessaire pour éviter une erreur judiciaire et lorsque le danger que la règle d'exclusion vise à prévenir n'existe pas.

24. R. c. Turpin, [1989] 1 R.C.S. 1296, p. 13091310.

25. R. c. Sherratt, [1991] 1 R.C.S. 509, p. 530531.

26. R. c. Find, [2001] 1 R.C.S. 863, par. 45.

27. Id., p. 533.

28. [1998] 1 R.C.S. 1128, par. 10.

29. C'est le cas d'un candidat ayant un lien quelconque avec l'un des intervenants dans le procès.
30. Ces attitudes et croyances peuvent découler de sa connaissance personnelle de l'affaire, de la publicité dans les médias ou de la discussion publique et de rumeurs dans la collectivité.

31. Les préjugés raciaux ou ethniques, ou les préjugés contre les personnes accusées d'abus sexuels, sont des exemples de préjugé générique.

32. [2001] 1 R.C.S. 863, par. 30 à 32 .

33. R. c. Sherratt, [1991] 1 R.C.S. 509, p. 532.

34. Chaque partie peut récuser sans motif un certain nombre de candidats jurés.

35. Cloutier c. La Reine, [1979] 2 R.C.S. 709, p. 720.

36. R. c. Bain, [1992] 1 R.C.S. 91, p. 153 (j. Stevenson, opinion minoritaire concordante)

37. Canada c. Tobiass, [1997] 3 R.C.S. 391, par. 103.

38. R. c. Find, [2001 1 R.C.S. 863, par. 41-42.

39. 2747-3174 Québec Inc. c. R.P.A.Q., [1996] 3 R.C.S. 919, par. 110-111 (j. L'Heureux-Dubé pour l'opinion minoritaire concordante).

40. L'alinéa 632c) du Code criminel dispose que le juge peut dispenser un juré pour toute raison valable qu'il considère acceptable, $\mathrm{y}$ compris un inconvénient personnel sérieux.

41. < http://www.cyberpresse.ca/>, consulté le 2002-08-17.

42. [1991] 1 R.C.S. 509, p. 525.

43. R. c. Parks, (1993) 84 C.C.C. (3d) 353, p. 364.

44. Les autochtones feraient notamment l'objet d'élimination en raison de leur race. Voir sur le sujet: Hamilton et Sinclair, 1991, p. 87; Casey, 1991.

45. [1992] 1 R.C.S. 91, p. 113. Les juges McLachlin et Iacobucci ont partagé l'opinion du juge Gonthier.

46. [1995] 1 R.C.S. 761.

47. Id., p. 788.

48. R. c. Corbett, [1988] 1 R.C.S. 670, p. 694.

49. Thompson Newspapers c. Canada (P.G.), [1998] 1 R.C.S. 877, par. 116.

50. R. c. Corbett, [1988] 1 R.C.S. 670, p. 692694.

51. R. c. Kuldip, [1990] 3 R.C.S. 618, p. 635.

52. R. c. Hibbert, [1995] 2 R.C.S. 973, par. 43.

53. R. c. Bain, [1992] 1 R.C.S. 91, p. 114.

54. L'article 633 du Code criminel dispose que le juge peut ordonner qu'un juré, dont le nom fut tiré au hasard, se tienne à l'écart «pour toute raison valable». 